\title{
CANCER
}

\section{A randomised study of screening for colorectal cancer using faecal occult blood testing: results after 13 years and seven biennial screening rounds}

\author{
O D Jørgensen, O Kronborg, C Fenger
}

See end of article for authors' affiliations

Correspondence to: Professor O Kronborg, Surgical Department A Odense University Hospital, DK-5000 Odense C, Denmark;

Ole.kronborg@dadlnet.dk

Accepted for publication 15 May 2001

\begin{abstract}
Background: Three randomised trials have demonstrated reduction in mortality from colorectal cancer $(C R C)$ by repeated screening with faecal occult blood tests, including the trial presented here, which is the only one still in progress.

Aims: To evaluate reduction in mortality after seven screening rounds and the possible influence of compliance on mortality from CRC.

Methods: At Funen in Denmark, random allocation to biennial screening with Hemoccult-ll in 30967 subjects aged 45-75 years and 30966 controls was performed in 1985 from a population of 137485 of the same age. Only participants who completed the first screening round were invited for further screening. Colonoscopy was offered if the test was positive. The primary end point was death from CRC, and the 10 year results were published in 1996.

Results: From the beginning of the first screening to the seventh round, mean age increased from 59.8 to 70.0 years in the screening and control groups, and the male/female ratio decreased from 0.92 to 0.81 . Those who accepted screening were younger than non-responders. Positivity rates varied from $0.8 \%$ to $3.8 \%$, the cumulative ratio of a positive test was $5.1 \%$ after seven rounds, and $4.8 \%$ of patients had at least one colonoscopy. Mortality from CRC was significantly less in the screening group (relative risk (RR) $0.82(0.69-0.97))$, and the reduction in mortality was most pronounced above the sigmoid colon. After seven rounds, RR was reduced to less than 0.70 compared with controls. Mortality rates from causes other than CRC did not differ. Non-responders had a significantly increased risk of death from CRC compared with those who accepted the full programme. Subjects who accepted the first screening, but not subsequent ones, demonstrated a tendency towards increased risk.

Conclusions: The persistent reduction in mortality from CRC in a biennial screening program with Hemoccult-II, and a reduction in RR to less than 0.70 in those adhering to the programme, support attempts to introduce larger scale population screening programmes. The smaller effect on mortality from CRC in the rectum and sigmoid colon suggests evaluation by additional flexible sigmoidoscopy with longer intervals.
\end{abstract}

repr hree randomised studies evaluating screening with Hemoccult-II (H-II), including the present study, have reported a significant reduction in mortality from colorectal cancer (CRC) ranging from $15 \%$ to $33 \%$ after 10 years, using annual or biennial screening. ${ }^{1-4}$ Screening of the average risk population above 50 years of age however has not yet been proved to be cost effective outside randomised trials.

We present the main results of biennial screening with H-II from 1985 to 1998 in a Danish study, which is still in progress, in contrast with the two other randomised studies, both of which are in the follow up phase. Causes of death have been updated since the publication of the 10 year results ${ }^{1}$ as well as values for compliance and possible influence on reduction in mortality from CRC.

\section{METHODS}

The design and randomisation procedure has been described in detail previously. ${ }^{1}$ In 1985, 61933 subjects, aged 45-75 years, were allocated randomly to either biennial screening with H-II $(n=30967)$ or to act as controls $(n=30$ 966). Subjects from the same household were allocated together. All subjects with known CRC, colorectal adenomas, or distant spread of all types of malignant disease were excluded before randomisation, which was possible using the public registers in Denmark.

Invitations were sent out by mail from the screening office. Two reminders were used during the initial screening round, and one during the following rounds. Only those participating in previous rounds and without CRC or adenomas were reinvited. Detection of CRC or adenomas resulted in invitation to a surveillance programme with colonoscopy. Each screening round was completed within one year.

Controls were not informed about the study, but events (CRC, adenoma, death) were registered in the same way as for those randomised to screening.

H-II was used without rehydration but with dietary restrictions. ${ }^{1}$ Subjects with a positive test were invited for interview, physical examination, and full colonoscopy. Double contrast barium enema (DCBE) was offered when complete colonoscopy could not be achieved or the patient did not want colonoscopy.

Information on new cases of CRC and adenomas was obtained throughout the study from the Funen County Database and the Danish National Registration of patients.

Death certificates were obtained from the county public health office and the central health authorities. Also, information on CRC and adenomas was cross checked with the Danish Cancer Registry. Death was certified as due to CRC according to criteria published previously. ${ }^{1}$ An independent

Abbreviations: H-II, Hemoccult-II; CRC, colorectal cancer; DCBE, double contrast barium enema; $R R$, relative risk. 
Table 1 Compliance during repeated screening

\begin{tabular}{lllll}
\hline & \multicolumn{2}{l}{ Screening group } & & Control group \\
\cline { 2 - 4 } $\begin{array}{l}\text { Screening } \\
\text { round }\end{array}$ & Subjects alive & $\begin{array}{l}\text { Subjects invited for } \\
\text { screening }\end{array}$ & Subjects screened (\%) & Subjects alive \\
\hline 1 & 30762 & 30762 & $20672(67)$ & 30966 \\
2 & 30100 & 20113 & $18781(93)$ & 30022 \\
3 & 28987 & 18236 & $17279(94)$ & 28882 \\
4 & 27742 & 16746 & $15845(94)$ & 27557 \\
5 & 26347 & 15279 & $14203(92)$ & 26227 \\
6 & 24798 & 13602 & $12533(92)$ & 24659 \\
7 & 23109 & 11902 & $11058(93)$ & 23028 \\
\hline
\end{tabular}

Table 2 Mean age and male/female ratio during repeated screening

\begin{tabular}{|c|c|c|c|c|c|c|c|c|}
\hline \multirow{3}{*}{$\begin{array}{l}\text { Screening } \\
\text { round }\end{array}$} & \multicolumn{6}{|c|}{ Screening group } & \multirow{2}{*}{\multicolumn{2}{|c|}{$\begin{array}{l}\text { Control group } \\
\text { Subjects alive }\end{array}$}} \\
\hline & \multicolumn{2}{|c|}{ Subjects alive } & \multicolumn{2}{|c|}{ Subjects invited } & \multicolumn{2}{|c|}{ Subjects screened } & & \\
\hline & Mean age & M/F ratio & Mean age & $M / F$ ratio & Mean age & $M / F$ ratio & Mean age & $\mathrm{M} / \mathrm{F}$ ratio \\
\hline 1 & 59.8 & 0.92 & 59.8 & 0.92 & 58.8 & 0.89 & 59.8 & 0.92 \\
\hline 2 & 61.6 & 0.91 & 60.7 & 0.87 & 60.5 & 0.87 & 61.7 & 0.91 \\
\hline 3 & 63.4 & 0.89 & 62.4 & 0.86 & 62.2 & 0.86 & 63.4 & 0.89 \\
\hline 4 & 65.2 & 0.87 & 64.0 & 0.85 & 63.8 & 0.86 & 65.1 & 0.87 \\
\hline 5 & 66.8 & 0.85 & 65.6 & 0.84 & 65.2 & 0.85 & 66.8 & 0.85 \\
\hline 6 & 68.5 & 0.84 & 67.1 & 0.83 & 66.6 & 0.85 & 68.5 & 0.83 \\
\hline 7 & 70.0 & 0.81 & 68.4 & 0.82 & 68.0 & 0.84 & 70.0 & 0.81 \\
\hline
\end{tabular}

review committee was consulted if it was not clear whether CRC was the cause of death.

In the present context, participants were followed until death or 1 August, 1998-that is, 13 years after initiation of the study. Incidence and mortality rates were calculated as the number of cases of CRC divided by the person years of observation.

Incidence and mortality ratios with 95\% confidence limits were obtained by Cox proportional hazards with the control group data as the denominator, unless otherwise stated. ${ }^{5}$ Models were compared using the likelihood ratio test. The Cox proportional hazards model with screening status included as a time dependent covariate was used to evaluate the effect of repeated screening. This strategy allowed for estimation of the risk of death after a specific number of screening rounds for those compliant with the screening programme relative to the risk in the control group in the corresponding time period. Similarly, the risk of death from CRC was estimated for patients who refused screening relative to those who accepted all screening rounds. Subjects were regarded as compliant with the screening programme as long as they did not refuse screening-that is, subjects in the screening group identified with adenomas or CRC (and therefore not invited to subsequent screening rounds) were regarded as compliant if they had not refused screening before the diagnosis.

The reduction in mortality from CRC proximal to the sigmoid colon was compared with that from distal CRC by Poisson regression. ${ }^{6}$

The study was approved by the regional ethics committee, and the population register was approved by the registry board of Funen.

\section{RESULTS}

The number of individuals who completed the H-II test at each screening round is shown in table 1. Two thirds accepted the initial screening, and only these subjects were reinvited to the following screening rounds. At the beginning of the last screening round, 23109 participants in the screening group were still alive. Of these, 11902 were invited to the latest screening round because they had participated in all previous screening rounds and had not been diagnosed with CRC or adenomas, except for 56 patients with adenomas unfit for adenoma surveillance, mainly because of age.

Mean age of those alive in the screening group increased from 59.8 years at the beginning of the first screening round to 70.0 years at the beginning of the latest screening round, and

Table 3 Further examination in subjects with a positive Hemoccult-II (H-II)

\begin{tabular}{|c|c|c|c|c|c|}
\hline $\begin{array}{l}\text { Screening } \\
\text { round }\end{array}$ & Positive H-II & $\begin{array}{l}\text { Complete } \\
\text { colonoscopy }\end{array}$ & $\begin{array}{l}\text { Incomplete } \\
\text { colonoscopy+ } \\
\text { DCBE }\end{array}$ & $\begin{array}{l}\text { Incomplete colonic } \\
\text { examination }\end{array}$ & $\begin{array}{l}\text { No colonic } \\
\text { examinations }\end{array}$ \\
\hline 1 & 215 (1.0) & $180(83.7)$ & 12 (5.5) & $17(7.9)$ & $6(2.9)$ \\
\hline 2 & 159 (0.8) & 142 (89.3) & 5 (3.1) & 7 (4.4) & $5(3.2)$ \\
\hline 3 & $151(0.9)$ & $124(82.1)$ & $15(9.9)$ & $8(5.2)$ & $4(2.8)$ \\
\hline 4 & 200 (1.3) & 168 (84.0) & 16 (8.0) & $10(5.0)$ & $6(3.0)$ \\
\hline 5 & $261(1.8)$ & $213(81.6)$ & $17(6.5)$ & $16(6.1)$ & $15(5.8)$ \\
\hline 6 & $478(3.8)$ & $394(82.4)$ & $21(4.3)$ & $19(3.9)$ & $44(4.6)$ \\
\hline 7 & 190 (1.7) & $159(83.6)$ & $13(6.8)$ & $5(2.6)$ & 13 (7.0) \\
\hline
\end{tabular}


Table 4 Incidence and mortality rates for colorectal cancer between 1985 and 1998

\begin{tabular}{|c|c|c|}
\hline & Screening & Controls \\
\hline \multicolumn{3}{|l|}{ Colorectal cancer } \\
\hline No patients & 649 & 637 \\
\hline Incidence rate* & 1.84 & 1.81 \\
\hline Incidence ratio & $1.02(0.91-1.14) \dagger$ & \\
\hline \multicolumn{3}{|l|}{ Death from all causes } \\
\hline No deaths & 8732 & 8724 \\
\hline Mortality rate* & 24.78 & 24.80 \\
\hline Mortality ratio & $1.00(1.00-1.00) \dagger$ & \\
\hline \multicolumn{3}{|l|}{ Death from colorectal cancer } \\
\hline No deaths & 255 & 310 \\
\hline Mortality rate* & 0.72 & 0.88 \\
\hline Mortality ratio & $0.82(0.69-0.97) \dagger$ & \\
\hline \multicolumn{3}{|l|}{ Death from colorectal cancer $\ddagger$} \\
\hline No deaths & 292 & 341 \\
\hline Mortality rate* & 0.83 & 0.97 \\
\hline Mortality ratio & $0.85(0.73-1.00) \dagger$ & \\
\hline $\begin{array}{l}\text { Mortality ratio adjusted for age } \\
\text { and sex }\end{array}$ & $0.86(0.73-1.00) \dagger$ & \\
\hline \multicolumn{3}{|c|}{ Death from colorectal cancer proximal to sigmoid colon $\ddagger$} \\
\hline No deaths & 89 & 123 \\
\hline Mortality rate* & 0.25 & 0.35 \\
\hline Mortality ratio & $0.72(0.55-0.95)$ & \\
\hline \multicolumn{3}{|c|}{ Deaths from distal colorectal cancer $\ddagger$} \\
\hline No deaths & 196 & 212 \\
\hline Mortality rate* & 0.56 & 0.60 \\
\hline Mortality ratio & $0.92(0.76-1.12)$ & \\
\hline
\end{tabular}

*Per 1000 person years; †95\% confidence limits; †including complications from treatment.

in the same period the male/female ratio decreased from 0.92 to 0.81 (table 2). Similar changes were observed among controls. In all screening rounds, the age of those who accepted the invitation to screening was less than the age of those who did not accept the invitation. More women than men accepted the invitation to the initial screening round but at the last four screening rounds more men accepted.

The proportion of individuals with a positive test varied from 0.8 to 3.8 , with a tendency to higher values at the last screenings (table 3 ). The cumulative risk of having a positive test was $5.1 \%$ ( 1559 of 30762 ). At least one colonoscopy was performed in $94.1 \%$ (1467 of 1559). The cumulative proportion of the whole screening group who underwent colonoscopy once or more during the screening period was therefore $4.8 \%$ ( 1467 of 30762 ).

A detailed description of further examinations in each screening round is presented in table 3; during the seven rounds, a complete colonoscopy was obtained in $81.6-89.3 \%$ of subjects with a positive H-II whereas complementary DCBE was necessary in 3.1-9.9\%. No further examination was performed in $2.8-7.0 \%$ - that is, 93 of 1559 positive tests $(5.9 \%)$.
Subjects were asked why they did not want to undergo further examinations and 39 gave no specific reason whereas 24 had accompanying severe diseases, and five had died from causes other than CRC before colonoscopy could be offered. Seven patients had bleeding haemorrhoids or fissure. Another seven persons had a diagnosis of CRC (six within two years of a positive $\mathrm{H}-\mathrm{II}$ ). The remaining 18 felt free of diseases or gave no rational explanation.

Of the 30762 individuals in the screening group, 22235 were alive on 1 August, 1998 (end of seventh round) compared with 22242 of the 30966 individuals in the control group. The incidence of CRC was similar in the two groups (table 4) but the mortality rate from CRC was significantly less in the screening group compared with the control group even when deaths from complications of treatment of CRC were included and adjustment made for age and sex (table 4). Screening was more effective $(p=0.04)$ in preventing death from CRC located proximal to the sigmoid colon than in preventing death from more distal CRC (table 4).

Mortality rates from all causes were identical in the screening group and controls, and mortality rates from causes other than CRC did not differ between the two groups. No mortality from colonoscopy itself appeared in the screening group.

Analysis of the subgroups revealed that the risk of death from CRC was significantly higher in men than in women (relative risk (RR) 1.33 (1.14-1.55)) but screening reduced the risk of death from CRC in a similar proportion of men and women $(p=0.94)$. The risk of death from CRC was also significantly higher among those aged 65 years or more compared with younger subjects (RR $1.73(1.60-1.87)$ ) but the effect of screening was independent of age $(p=0.28)$.

The effect of compliance with the screening programme is shown in table 5 . After seven screening rounds, the relative risk of death from CRC was reduced to less than 0.70 compared with the risk in the control group in the corresponding observation time. Patients who refused any screening had a significantly increased risk of death from CRC compared with those who accepted the full screening programme. Also, subjects who refused subsequent screenings after the initial one showed a non-significant tendency towards an increased risk.

\section{DISCUSSION}

The present screening programme has shown that the risk of death from CRC was reduced to $0.85(0.73-1.00)$ relative to the risk among controls after 13 years and seven screening rounds. This is a decrease in benefit compared with the results after 10 years and five screening rounds where a relative risk of $0.82(0.68-0.99)$ was reported. ${ }^{2}$ The trend can be attributed mainly to the decreasing proportion of the screening group actually being screened, and is not caused by increasing age or changes in the male/female ratio as one might expect (to date, age and sex have not influenced the effect of screening).

Table 5 Risk of death from colorectal cancer according to number of screening rounds for subjects who refused screening and for subjects who adhered to the screening programme, respectively

\begin{tabular}{lllll}
\hline $\begin{array}{l}\text { Screening } \\
\text { round }\end{array}$ & $\begin{array}{l}\text { Subjects } \\
\text { screened }\end{array}$ & $\begin{array}{l}\text { Relative risk after } \\
\text { screening rounds }\end{array}$ & $\begin{array}{l}\text { Subjects refusing } \\
\text { screening at this or } \\
\text { subsequent rounds }\end{array}$ & Relative risk* \\
\hline 1 & 20672 & $0.70(0.58-0.85)$ & 16110 & $1.65(1.30-2.08)$ \\
2 & 18781 & $0.69(0.57-0.84)$ & 6060 & $1.34(0.94-1.99)$ \\
3 & 17279 & $0.66(0.54-0.81)$ & 4744 & $1.36(0.88-2.11)$ \\
4 & 15845 & $0.67(0.55-0.82)$ & 3799 & $0.97(0.53-1.77)$ \\
5 & 14203 & $0.67(0.55-0.82)$ & 2912 & $1.31(0.68-2.53)$ \\
6 & 12533 & $0.67(0.53-0.81)$ & 1860 & No events \\
7 & 11058 & $0.66(0.54-0.81)$ & 820 & \\
\hline & * Relative to subjects accepting all screening rounds. & & \\
\hline
\end{tabular}


Re-invitation of all those in the screening group, and not only subjects who participated in earlier screening rounds, may have improved the long term effect of the screening programme by preventing a decrease in the participation rate. On the other hand, the experience of others suggests that the effect of inviting those who once refused screening is relatively small. $^{2}$

Reduction of the relative risk of death from CRC to less than 0.70 for subjects adhering to the screening programme highlights the benefit of repeated screening. It should be emphasised that subjects adhering to the screening programme included those identified with CRC and adenomas who accepted all screening invitations previous to the diagnosis. Subjects who refused screening after participation in one or more screening rounds seemed to have an increased risk of death from CRC compared with those who adhered to the screening programme but this was not statistically significant. Colonoscopy was accepted in the majority of those with a positive test, and the explanation for not undergoing colonoscopy in the $5.9 \%$ of patients who refused unfortunately gave no guidance for a change in the present strategy. Overall, death rates from diseases other than CRC did not increase significantly in the screening group compared with controls.

Most of the observations made to date favour a screening programme with at least seven biennial screening rounds, although the increasing positivity rate and rising number of colonoscopies may increase the economic cost of the programme as well as the inconvenience and risk for the participants. However, another screening programme, reporting a reduction in mortality from CRC of $21 \%$ using rehydrated $\mathrm{H}$-II, had a positivity rate of almost $10 \%$ and a cumulative rate of colonoscopy of $27 \%$ after 13 years of biennial screening, which is much higher than our values. ${ }^{3}{ }^{4}$ The Minnesota study however was performed in volunteers in contrast with the European true population studies, making it difficult to draw valid comparisons.

Our screening programme with $\mathrm{H}$-II resulted in an $8 \%$ reduction in the risk of death from CRC located in the sigmoid colon and rectum compared with a reduction of $28 \%$ for patients with CRC in the remaining colon. These results support a screening programme using a combination of flexible sigmoidoscopy and H-II. Reduction in death from CRC in the rectum and sigmoid colon by screening with flexible sigmoidoscopy has been demonstrated only in case control studies. ${ }^{7}$ The Nottingham study ${ }^{2}$ reported mortality ratios of $0.87(0.68-1.11)$ for cancer proximal to the sigmoid colon and $0.84(0.70-1.00)$ for distal cancers, in contrast with our findings, whereas no information is available from the Minnesota study. ${ }^{3}$ Our findings suggest that a randomised study evaluating the possible influence on reduction in mortality from CRC by adding flexible sigmoidoscopy to faecal occult blood testing is warranted.

It was thought that the more frequent removal of large colorectal adenomas in the screening group might lead to a decrease in the incidence of CRC, according to the adenomacarcinoma sequence. ${ }^{8}$ This has not happened to date, although other pathways do exist. ${ }^{9}$ However, a decrease in incidence of $>17 \%$ after an 18 year follow up period was reported recently from the Minnesota group. ${ }^{10}$ Accordingly, the present study will continue to further assess compliance for repeated screenings and the possible effect of increasing age, as well as the influence of screening on the incidence of CRC. The eighth screening round was completed in 1999 but data have not yet been analysed, and the ninth screening round began in August 2001.

\section{Authors' affiliations}

O D Jørgensen, O Kronborg, Department of Surgical

Gastroenterology, Odense University Hospital, Denmark

C Fenger, Department of Pathology, Odense University Hospital, Denmark

\section{REFERENCES}

1 Kronborg O, Fenger C, Olsen J, et al. Randomised study of screening for colorectal cancer with faecal-occult-blood test. Lancet 1996:348:1467-71.

2 Hardcastle JD, Chamberlain JD, Robinson MHE, et al. Randomised controlled trial of faecal-occult-blood screening for colorectal cancer Lancet 1996;348:1472-7.

3 Mandel JS, Bond JH, Church TR, et al. For the Minnesota Colon Cancer Control Study. Reducing mortality from colorectal cancer by screening for fecal occult blood. N Engl J Med 1993;328:1365-71.

4 Mandel JS, Church TR, Ederer F, et al. Colorectal cancer mortality: Effectiveness of biennial screening for fecal occult blood. J Natl Cancer Inst 1999;91:434-7.

5 Hopkins A. Survival analysis with covariates-Cox models. In: Dixon WJ, ed. BMDP statistical software manual. Berkeley, California: University of California Press, 1988:941-68.

6 Breslow NE, Day NE. Statistical methods in cancer research. The design and analysis of cohort studies. Lyon: IARC, 1987.

7 Selby JV, Friedman GD, Quesenberry CP, et al. A case-control study of screening sigmoidoscopy and mortality from colorectal cancer. N Engl J Med 1992; 326:653-7

8 Vogelstein B, Fearon ER, Hamilton SR, et al. Genetic alterations during colorectal tumor development. N Engl J Med 1988;319:525-32.

9 Ilyas M, Straub J, Tomlison IPM, et al. Genetic pathways in colorectal and other cancers. Eur J Cancer 1999;35:335-51.

10 Mandel JS, Church TR, Bond JH, et al. The effect of fecal occult-blood screening on the incidence of colorectal cancer. N Engl J Med 2000;343: 1603-7. 